vessel. The tritium doubling time would perhaps be of the order of one year. The reactor would also be a very fruitful source of neutrons, but would produce far less radioactive waste than fission reactors. More recent calculations, Professor Rose says, have shown that fusion reactors would produce one ten thousandth as much overall radioactivity as fission reactors of comparable size. Another advantage is that when fusion reactors are shut down, the total afterheat would be tiny - something like 60 kilowatts for a reactor: of 10,000 to $15,000 \mathrm{MW}$. A large fission reactor, by contrast, may be nominally shut down by insertion of the control rods, but nevertheless goes on producing very large quantities of heat-perhaps 10,000 times as much as the fusion reactor the same size.

One of the important results of Professor Rose's report (apart from sustaining optimism among plasma physicists, which is itself important) may be to redirect some of the research effort. Professor Rose is particularly keen that more work should be done on the effects of the energetic neutrons on the reactor vessel. There is no experience of radiation damage at these extreme energies. There is also the question of injecting fuel into the reactor, which may turn out to be a great deal harder than it sounds. But Professor Rose concludes that a fusion power station, if feasible, would be cheap. Capital costs in the region of $£ 12$ per kilowatt and a generating cost of 0.3 pence per kilowatt look good. By the year 2000, of course, there is always the chance that they will have been overtaken by something even better.

\section{Steady State Supported}

THE steady state theory of cosmology still has a great deal of life in it, according to Professor Fred Hoyle, director of the Institute of Theoretical Astronomy at the University of Cambridge. Professor Hoyle, giving this year's Bakerian Lecture at the Royal Society last week, discussed how the evidence put forward against the steady state theory can be overcome. He emphasized, however, that his lecture - a review of recent. developmonts in cosmology - was an entirely personal view of the subject. His interpretation of counts of radio sources, for example, certainly differs from that of several other astronomers.

The most recent challenge to the steady state theory, Professor Hoyle said, was the discovery about three years ago of the microwave background radiation. This is radiation at microwave frequencies which seems to permeate space and corresponds to a black-body temperature of about $3^{\circ} \mathrm{K}$. One school of thought believes the microwave radiation is a by-product of events early in the history of a so-called big-bang universe. One of the reasons for this interpretation is the difficulty in understanding the radiation in terms of emission from existing radio sources which are not expected to emit strongly enough at short wavelengths to account for the measured intensities. The microwave background has consequently been widely held to be a fossil relic of a big-bang cosmology. Professor Hoyle said, however, that there is evidence coming to light that the spectra of an appreciable fraction of radio sources have an upturn toward higher frequencies. This means the microwave background may yet be explained by emission from radio sources, and that the steady state theory will be upheld in this respect.
In his lecture, Professor Hoyle went through the crises the steady state theory had so far faced. In particular, he discussed the way in which counts of radio sources can distinguish between different cosmologies. The practice is to count the number of radio sources $N$ brighter than a certain flux $S$, and to plot $\log N$ against $\log S$. Various cosmologies predict different values for the slope of the straight line through the points, the steady state theory predicting a slope of -1.5 , for instance. Professor Hoyle said that counts of radio sources in the Cambridge $4 C$ and $5 C^{\prime}$ surveys agree with the steadr state cosmology. but that the earlier $3 C$ survey gives a steeper slope more in keeping with the big-bang theory. To understand the steep slope given by the $3 C$ survey in terms of the steady state cosmology, the radio sources making up the surver can be classified according to their appearance and the slope of the $\log N$-log $S$ curve evaluated for each class. Objects which look like radio galaxies lead to a slope of $-1 \cdot 4$. and sources identified as quasars have a slope of -1.56 . The remainder of sources in the $3 C^{r}$ survey, which could not definitely be identified as quasars or as radio galaxies, contributed a slope of $-2 \cdot 5$. Professor Hoyle says the disagreement of the $3 C$ surver with the steady state theorv can thus be attributed to the contribution to the counts of sources the nature of which is unknown, and which therefore may have no cosmological significance. New counts of radio sources now under way in the southern hemisphere may help to resolve this question.

Another criterion for distinguishing cosmologies involves the red-shift of distant objects. The technique is to compare the observed relation between red-shift. and visual magnitude with the relation expected for various cosmologies. All cosmologies predict the same relation between red-shift and visual magnitude for comparatively local objects, however, and it was not until the discovery of the quasars, with their large red-shifts, that red-shifts could be used by cosmologists in this way. Although measurements of quasars tend to support the big-bang theory, there is doubt whether their large red-shifts are in fact of cosmological significance at all. The problem seerns to hinge around indications that quasars are large masses confined in volumes comparatively small by astronomical standards. This suggests that their large red-shifts may be a gravitational effect, yet the spectra of quasars are not what are expected for emission in a high gravita. tional field. Further, some quasars seem to show more than one red-shift, and up to nine red-shifts have been associated with one object. This may mean the emission is coming from differing positions in a strong gravitational field, or an alternative explanation is that the quasar is ejecting clouds of gas at speeds approaching the speed of light. The uncertainty about what quasars really are makes their cosmological significance much less convincing.

\section{Daresbury's Plan}

The Nuclear Physics Laboratory at Daresbury sees as one of its functions the design of accelerators which may form the future development of the sitc. At the moment, for example, the Daresbury laboratory is completing a design study for an electron synchrotron producing energies which may go up to $20 \mathrm{GeV}$. One of the features of the Daresbury design is that it uses 